\section{OPEN ACCESS}

Edited by:

Li Ang,

First Affiliated Hospital of Zhengzhou

University, China

Reviewed by:

Ping Ma,

Tianjin Second People's

Hospital, China

Citra Fragrantia Theodorea,

University of Indonesia, Indonesia

*Correspondence:

Zhiliang Hu

huzhiliang@njucm.edu.cn

Hongxia Wei

wghongxia@sina.com

tThese authors have contributed equally to this work

Specialty section:

This article was submitted to Infectious Diseases - Surveillance,

Prevention and Treatment

a section of the journa

Frontiers in Public Health

Received: 08 July 2021 Accepted: 03 December 2021

Published: 21 January 2022

Citation:

Zhu Y, Xu M, Ding C, Peng Z, Wang W, Sun B, Cheng J, Chen C, Chen W,

Wei $\mathrm{H}$ and $\mathrm{Hu} Z$ (2022) Metagenomic Next-Generation Sequencing vs.

Traditional Microbiological Tests for Diagnosing Varicella-Zoster Virus Central Nervous System Infection.

Front. Public Health 9:738412. doi: 10.3389/fpubh.2021.738412

\title{
Metagenomic Next-Generation Sequencing vs. Traditional Microbiological Tests for Diagnosing Varicella-Zoster Virus Central Nervous System Infection
}

Yunqi Zhu ${ }^{1+}$, Miaomiao $\mathrm{Xu}^{1+}$, Chengyuan Ding $^{2+}$, Zhihang Peng ${ }^{3}$, Weixiao Wang ${ }^{4}$, Binghu Sun ${ }^{1}$, Jian Cheng ${ }^{1}$, Chen Chen ${ }^{1}$, Wei Chen ${ }^{4}$, Hongxia Wei ${ }^{1 *}$ and Zhiliang $\mathrm{Hu}^{1,2 *}$

${ }^{1}$ Department of Infectious Disease, The Second Hospital of Nanjing, Nanjing University of Chinese Medicine, Nanjing, China, ${ }^{2}$ Center for Global Health, School of Public Health, Nanjing Medical University, Nanjing, China, ${ }^{3}$ School of Public Health, Nanjing Medical University, Nanjing, China, ${ }^{4}$ Department of Clinical Research Center, The Second Hospital of Nanjing, Nanjing University of Chinese Medicine, Nanjing, China

Background: Unbiased metagenomic next-generation sequencing (mNGS) detects pathogens in a target-independent manner. It is not well-understood whether mNGS has comparable sensitivity to target-dependent nucleic acid test for pathogen identification.

Methods: This study included 31 patients with chickenpox and neurological symptoms for screening of possible varicella-zoster virus (VZV) central nervous system (CNS) infection. Microbiological diagnosing of VZV cerebrospinal fluid (CSF) infection was performed on stored CSF samples using mNGS, quantitative and qualitative VZV-specific PCR assays, and VZV IgM antibodies test.

Results: The median age was 30.0 [interquartile range (IQR), 24.3-33.3] years. 51.6\% of the patients were men. About $80.6 \%$ of the patients had normal CSF white blood cell counts $\left(\leq 5 \times 10^{6} / \mathrm{L}\right)$. VZV IgM antibodies presented in $16.1 \%$ of the CSF samples, and nucleic acids were detectable in 16.1 and $9.7 \%$ using two different VZV-specific real-time PCR protocols. Intriguingly, maximal identification of VZV elements was achieved by CSF mNGS $(p=0.001$ and $p=007$; compared with qualitative PCR and VZV IgM antibody test, respectively), with sequence reads of VZV being reported in $51.6 \%(16 / 31)$ of the CSF samples. All VZV PCR positive samples were positive when analyzed by mNGS. Of note, human betaherpesvirus $6 \mathrm{~A}$ with clinical significance was unexpectedly detected in one CSF sample.

Conclusions: Our study suggests that CSF mNGS may have higher sensitivity for VZV detection than CSF VZV PCR and antibody tests, and has the advantage of identifying unexpected pathogens.

Keywords: metagenomic next-generation sequencing, varicella-zoster virus, central nervous system infection, polymerase chain reaction, nucleic acid test 


\section{INTRODUCTION}

Central nervous system (CNS) infections have led to a substantial morbidity and mortality in clinical practice; however, the etiologic agents remain undetermined in around half of the cases $(1,2)$. The diagnostic challenge is that more than 100 infectious agents can cause encephalitis, and that the initial neurological manifestations of many CNS infections are nonspecific (3-5). It is difficult to measure all the CNS pathogens by traditional microbiological tests, such as morphological, serological, molecular, and culture methods (5). An acceptable, but not optimal, strategy is to target the most possibly causative pathogens based on a clinical judgment. However, in this context, the efficacy of pathogen discovery would largely depend on the clinical experience of the treating physician. Moreover, the etiologies of cases with atypical manifestations or caused by rare pathogens are likely to be unconfirmed.

Unlike the traditional pathogen-detection methods, which require a prior knowledge of the causative agents, the unbiased metagenomic next-generation sequencing (mNGS) identifies pathogens in a target-independent way (6). It has been reported that cerebrospinal fluid (CSF) mNGS helps to detect known as well as unexpected CNS pathogens (7-11). However, it is less clear whether mNGS has comparable sensitivity as compared with traditional pathogen-detection methods for diagnosing a specific CNS infection. Clinicians may still want to know whether pathogen-specific nucleic acid test is needed when an $\mathrm{mNGS}$ reveals a negative result for the pathogen of interesting. For the diagnosis of CNS Mycobacterium tuberculosis (MTB) infection, studies have shown that mNGS has higher sensitivity as compared with MTB-specific PCR $(12,13)$. Whether similar finding could be found in viral encephalitis needs to be further studied.

The sensitivity of CSF mNGS for pathogen detection may be greatly influenced by the CSF pleocytosis which leads to a substantial human DNA contamination (14). Since viral encephalitis generally has less inflamed CSF compared with other forms of CNS infections, the advantage of pathogen identification by mNGS may be more prominent in viral encephalitis. In the present study, we aimed to investigate the potency of CSF mNGS for identification of the etiologic agent using a model of varicella-zoster virus (VZV) CNS infection.

\section{METHODS}

\section{Patients}

This study included 31 patients with suspected VZV CNS infection admitted to the second hospital of Nanjing, China, from August 2018 to November 2020. In Nanjing, the second hospital of Nanjing is the only designated hospital for managing patients with primary VZV infection. Patients with characteristic skin rashes suggestive of varicella and concomitant neurological symptoms were hospitalized for screening of possible VZV CNS involvement. Diagnostic procedures in routine clinical care for those patients included a cerebral MRI and analysis of CSF cell counts, chemistry, and bacterial culture. After obtained written informed consent, additional CSF samples were collected and stored at $-80^{\circ} \mathrm{C}$ until analysis for VZV IgM antibodies, VZV PCR, and unbiased mNGS.

The medical records, such as epidemiological and demographic data, medical history, underlying comorbidities, clinical manifestations, laboratory tests, cerebral imaging findings, treatments, and outcomes, were retrieved from an electronic health record system. This study was approved by the ethics committee of the second hospital of Nanjing (reference number: 2020-LY-kt061).

\section{Metagenomic Next-Generation Sequencing}

Cerebrospinal fluid samples were sent for PMSeq-DNA, a commercially available service from BGI-Shenzhen, which detects pathogenic microorganisms through mNGS. In this study, $600 \mu \mathrm{l}$ CSF sample was mixed with enzyme and glass beads, and was vortexed vigorously at 2,800-3,200 rpm for $30 \mathrm{~min}$. Then, total DNA was extracted from $300 \mu \mathrm{l}$ each CSF sample using TIANamp Micro DNA Kit (DP316, Tiangen Biotech, Beijing, China) according to the recommendation of manufacturer. DNA libraries were constructed through DNA-fragmentation, end-repair, adapter-ligation, and PCR amplification. After quality control with Agilent 2100 (Agilent Technologies, CA, USA), libraries were sequenced by BGISEQ50 platform. Low-quality sequence reads and sequence reads mapped to the human reference genome (hg19) were removed. The remaining data were classified by aligning to four Microbial Genome Databases containing bacteria, fungi, viruses, and parasites. The reference databases were downloaded from NCBI (ftp://ftp.ncbi.nlm.nih.gov/genomes/) which contained 4,945 whole genome sequences of viral taxa, 6,350 bacterial genomes or scaffolds, 1,064 pathogenic fungi genomes, and sequences of 234 parasites associated with human diseases.

\section{Detection of VZV-IgM Antibodies}

An ELISA kit (ESR104M, SERION, Friedrich-Bergius-Ring, Würzburg, Germany) was used to measure VZV-IgM antibodies in CSF samples. The ELISA procedure was done following SERION ELISA classic CSF Diagnostics instructions with a modification that the dilution factor of CSF samples was changed from 1:20 to 1:10. As a CSF standard was not included in the kit, the interpretation of ELISA results was based on average optical density (OD) 450 values. The ELISA test was considered negative if the average OD value from duplicate sample wells is $<0.1$ or was $<2$-fold of that in the negative control. In our study, CSF samples from nine patients with syphilis-seropositive were used as negative control. Those nine patients were excluded from neurosyphilis based on normal CSF cell counts and chemistry tests, and negative CSF treponema pallidum particle agglutination assays. The ELISA assay was considered positive if the average OD value from each of the duplicate sample wells was $\geq 2$-fold of that in the negative control.

\section{Real-Time PCR Detection Assay}

The VZV nucleic acid was detected by real-time PCR assay. For the qualitative assay, $30 \mu \mathrm{l}$ of total DNA was extracted from $200 \mu \mathrm{l}$ CSF sample using TIANamp Micro DNA Kit (DP316, 
Tiangen Biotech). Then, the presence of VZV DNA was detected using a commercially available VZV nucleic acid detection kit following the instruction of manufacturer (D2211, Daan Gene, Guangdong, China). Each sample was testes two times. The following PCR program was run in the applied biosystems (ABI) 7500 Thermal Cycler (Applied Biosystems, MA, USA): $50^{\circ} \mathrm{C}$ for $2 \mathrm{~min}$; $95^{\circ} \mathrm{C}$ for $15 \mathrm{~min}$; 45 cycles of $94^{\circ} \mathrm{C}$ for $15 \mathrm{~s}$; and $55^{\circ} \mathrm{C}$ for $45 \mathrm{~s}$. A cycle threshold $(\mathrm{Ct})$ value of 38 or less was considered positive.

In addition, the CSF samples were sent for absolute quantification of the VZV viral load using an in-house realtime quantification PCR test at a third-party diagnostic company (New Jingpei Diagnostics, Shanghai, China). Then, $100 \mu \mathrm{l}$ of total DNA was extracted from $200 \mu \mathrm{l}$ CSF sample using a DNA/RNA extraction Kit (ZTLJB, Tianlong Biotech, Hangzhou, China). Furthermore, $5 \mu \mathrm{l}$ DNA sample was added to the reaction mix prepared following instruction of QuantiTect Probe PCR Kit (204343, Qiagen, Germany). The primers and probe set for VZV DNA amplification and detection were as follows: forward primer: 5'-TGCAGGGCATGGCTCAGT-3'; reverse primer: 5' CCCAAGAACCACATGTCCAAC-3'; and probe: $5^{\prime}$-FAM-TCC TCAGTGCGGTGGTTGCCCA-BHQ1-3' (15). The following PCR program was run in the applied biosystems (ABI) 7500 Thermal Cycler: $50^{\circ} \mathrm{C}$ for $2 \mathrm{~min} ; 95^{\circ} \mathrm{C}$ for $15 \mathrm{~min}$; 45 cycles of $94^{\circ} \mathrm{C}$ for $15 \mathrm{~s}$; and $60^{\circ} \mathrm{C}$ for $40 \mathrm{~s}$. Standard curve for absolute quantification was generated by amplifying serial dilutions of a plasmid containing VZV DNA segment. The lower limit of detection as evaluated by the service provider was 500 copies $/ \mathrm{ml}$.

\section{Statistical Analysis}

Continuous variables were expressed as the medians and interquartile ranges (IQRs). Categorical variables were shown as the counts and percentages in each category. Comparison between groups was done using McNemar's test for categorical variables, when appropriate. Statistical analysis was done by using SPSS version 22.0 (IBM, NY, USA). A $p<0.05$ is considered statistically significant.

\section{RESULTS}

\section{The Characteristics of the Patients}

The characteristics of the 31 patients admitted for screening of the VZV CNS infection were summarized in Table 1. None of the patients previously received chickenpox vaccine or had any evidence of immune deficiency. The diagnosis of primary VZV infection was mainly based on the presence of typical rashes morphologically resembling chickenpox. Serum VZV IgM antibodies were positive in $66.7 \%(8 / 12)$ of the patients. The median age was 30.0 (IQR, 24.3-33.3) years. About 51.6\% (16/31) of the patients were men, and $54.8 \%$ (17/31) patients had contacted with chickenpox within 4 weeks. The median time from onset of illness to admission was 4.0 (3.0-5.0) days, while from onset of neurological symptoms was 2.0 (1.0-4.0) days. The most common neurological symptom was headache $(93.5 \%$, $29 / 31)$, followed by nausea $(45.2 \%, 14 / 31)$ and vomiting $(19.4 \%$, $6 / 31)$. Signs of meningeal irritation were not common, with nuchal rigidity seen in $12.9 \%(4 / 31)$ of the patients and Kernig's
TABLE 1 | Clinical, laboratory, and imaging characteristics of the 31 patients hospitalized for screening of varicella-zoster virus (VZV) central nervous system (CNS) infection.

\begin{tabular}{|c|c|}
\hline & Total $(n=31)$ \\
\hline \multicolumn{2}{|l|}{ Demographics and clinical characteristics } \\
\hline Age, years & $30(24.3-33.3)$ \\
\hline Sex, male & $16(51.6)$ \\
\hline Comorbidity & $2(6.5)$ \\
\hline Recently contacted with chickenpox & $17(54.8)$ \\
\hline Time from chickenpox onset, days & $4.0(3.0-5.0)$ \\
\hline Time from neurological symptoms onset, days & $2(1-4)$ \\
\hline Headache & $29(93.5)$ \\
\hline Neuasia & $14(45.2)$ \\
\hline Vomitting & $6(19.4)$ \\
\hline Mental Change & $2(6.5)$ \\
\hline Difficulty urinating & $2(6.5)$ \\
\hline Nuchal rigidity & $4(12.9)$ \\
\hline Kernig's sign & $1(3.2)$ \\
\hline Brudzin ski’s sign. & $0(0)$ \\
\hline \multicolumn{2}{|l|}{ Blood laboratory tests } \\
\hline White blood cell count, $\times 10^{9} / \mathrm{L}$ & $4.9(3.9-7.0)$ \\
\hline Lymphocyte count, $\times 10^{9} / \mathrm{L}$ & $1.3(1.1-2.9)$ \\
\hline Lactate dehydrogenase, IU/L & $240.0(202.5-289.5)$ \\
\hline C-reactive protein, mg/L & $10.8(2.7-20.9)$ \\
\hline \multicolumn{2}{|l|}{ Cerebrospinal fluid analysis } \\
\hline White blood cell count, $\times 10^{6} / \mathrm{L}$ & $2.0(1.0-5.0)$ \\
\hline Proteins, mg/L & $261.4(219.7-354.8)$ \\
\hline Glucose, mmol/L & $3.2(2.9-3.8)$ \\
\hline Lactate dehydrogenase, IU/L & $17(14-21)$ \\
\hline Adenosine deaminase & $2(0.1-3.6)$ \\
\hline Negative bacterial culture & $31(100)$ \\
\hline \multicolumn{2}{|l|}{ Brain imaging } \\
\hline Abnormal magnetic resonance imaging & $4(12.9)$ \\
\hline
\end{tabular}

sign in $3.2 \%(1 / 31)$ of the patient. The majority of the patients $(80.6 \%, 25 / 31)$ had CSF white blood cell counts not more than 5 $\times 10^{6}$ cells/L.

\section{Diagnosis of VZV CNS Infection Through CSF VZV IgM Detection, VZV PCR, and mNGS}

At the time of discharge, $41.9 \%(13 / 31)$ of the patients were clinically diagnosed with VZV CNS infection. Of the 13 patients with a clinical diagnosis of VZV CNS infection, 6 had CSF pleocytosis (leukocytes $>5 \times 10^{6}$ cells/L). The diagnosis of VZV CNS infection in the remaining 7 patients with normal CSF white blood cell counts was made by expert penal after thoroughly evaluating the clinical course and imaging findings of those patients. To explore the microbiological evidence of VZV CNS infection, stored CSF samples were tested for VZV IgM antibodies, VZV PCR, and unbiased mNGS. VZV IgM antibodies were presented in $16.1 \%(5 / 31)$ of the CSF samples. 
TABLE 2 | Diagnosis of VZV CNS infection.

\begin{tabular}{lcc}
\hline & \multicolumn{2}{c}{ CSF mNGS } \\
\cline { 2 - 3 } & $\begin{array}{c}\text { VZV detected } \\
(\boldsymbol{n}=\mathbf{1 6})\end{array}$ & $\begin{array}{c}\text { VZV not detected } \\
(\boldsymbol{n}=\mathbf{1 5})\end{array}$ \\
\hline $\begin{array}{l}\text { Clinical diagnosed VZV CNS } \\
\text { infection }\end{array}$ & $7(43.8)$ & $6(40.0 \%)$ \\
$\begin{array}{l}\text { Positive CSF VZV IgM antibodies } \\
\text { CSF VZV nucleic acid test }\end{array}$ & $3(18.8)$ & $2(13.3)$ \\
$\begin{array}{l}\text { (qualitatively) } \\
\text { CSF VZV nucleic acid test } \\
\text { (quantitatively) }\end{array}$ & $5(31.5)$ & $0(0)$ \\
Abnormal cerebral MRI & $3(18.8)$ & $0(0)$ \\
\hline
\end{tabular}

CSF, cerebrospinal fluid; mNGS, metagenome next-generation sequencing; VZV, varicella-zoster virus; CNS, central nervous system; MRI, magnetic resonance imaging. ${ }^{a}$ The median [interquartile range (IQR)] sequence reads of CSF mNGS is 34.6 (28.848.3) millions.

Varicella-zoster virus nucleic acids were detected in $16.1 \%$ $(5 / 31)$ and $9.7 \%(3 / 31)$ of the CSF samples, respectively, when different real-time PCR reagents were used. VZV nucleic acids were detected in three CSF samples by both qualitative and quantitative VZV PCR. Two CSF samples, with negative quantitative VZV PCR results, tested weak positive for VZV by using qualitative VZV PCR with cycle threshold of 37.88 and 37.93 , respectively. Intriguingly, maximal identification of VZV elements was achieved by CSF mNGS $(p=0.001$ and $p=007$; compared with qualitative PCR and VZV IgM antibody test, respectively), with sequence reads of VZV being reported in $51.6 \%(16 / 31)$ of the CSF samples (Table 2). The VZV relative abundance was provided in Supplementary Table 1. All the cases with positive CSF VZV PCR results tested positive for VZV sequence reads with CSF mNGS (Table 2).

\section{Pathogens Unexpectedly Identified by mNGS and the Clinical Significance}

Of the 16 patients with VZV DNA detected by mNGS, the median VZV read was 6 (IQR, 1-85) (Figure 1A); VZV was the only reported pathogen in five CSF samples, and the remaining 11 CSF samples also detected other pathogens, most commonly the CMV (5 CSF samples; range of sequence reads, 1-26) (Figure 1B). Pathogens unexpectedly identified by mNGS in the 31 CSF samples were shown in Table 3; of which, 5 had HSV1 sequence reads (range, 1-155). The presence of CMV and HSV-1 was evaluated by two real-time PCR based kits (Sansure Biotech, Hunan, China), and none of the CSF sample showed positive result.

A mNGS of the CSF sample revealed relatively high sequence reads of human betaherpesvirus $6 \mathrm{~A}$ (HHV-6A) and low sequence reads of VZV ( 889 and 6 , respectively). The patient was admitted to the hospital because of fever and itchy blisters for 6 days and difficulty urinating for 1 day. She had a close contact with her son who was recently diagnosed with chickenpox. CSF analysis demonstrated pleocytosis with leukocytes of $120 \times 10^{6}$ cells/L. A cerebral MRI showed multiple abnormalities in left thalamus, cerebellar vermis, and left cerebral peduncles. At that time, she was clinically diagnosed with VZV CNS infection, and
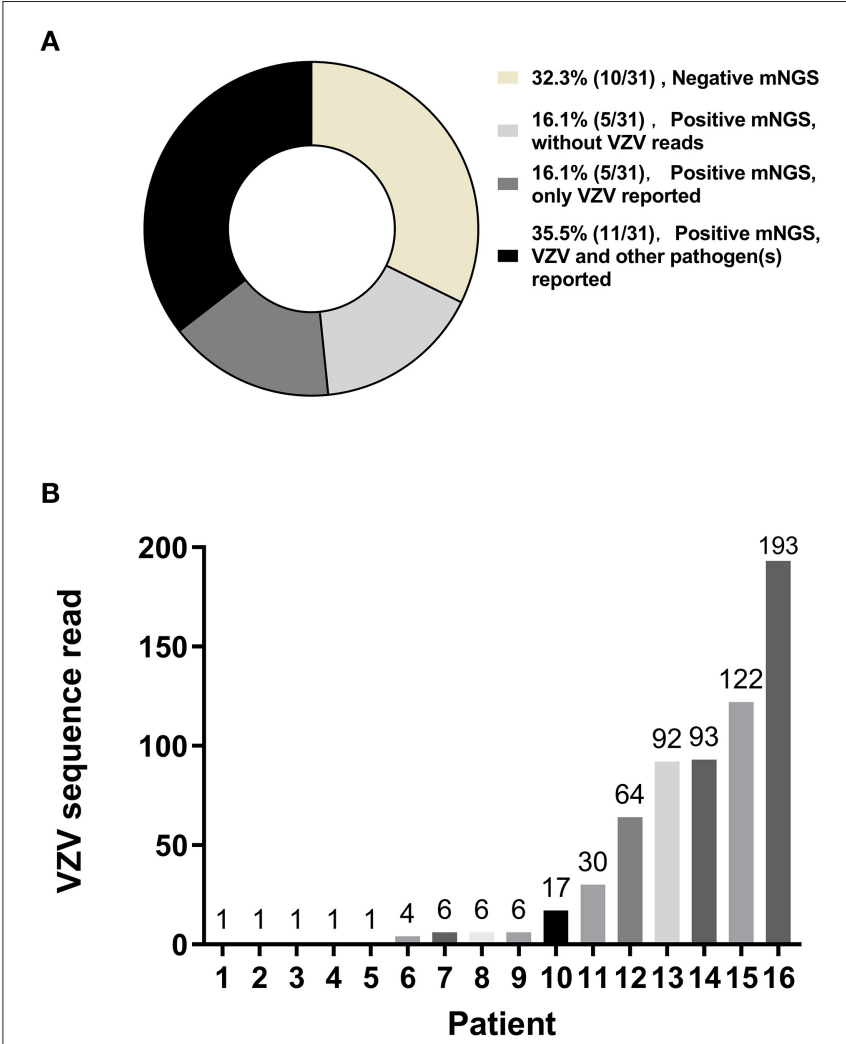

FIGURE 1 | Metagenomic next-generation sequencing (mNGS) of cerebrospinal fluid (CSF) samples. Metagenomic next-generation sequencing was performed on CSF samples from 31 patients with suspected varicella-zoster virus (VZV) central nervous system (CNS) infections. VZV sequence reads were the only sequence reads reported in $16.1 \%(5 / 31)$ of the patients. VZV sequence reads and sequences reads of other pathogens were both reported in $35.5 \%(11 / 31)$ of the patients (A). Of those 16 patients with VZV being detected by CSF mNGS, variable numbers of VZV sequence reads were reported $\mathbf{( B )}$.

was treated with acyclovir, intravenous immunoglobulin, and dexamethasone. The clinical condition was gradually improved. After hospitalization for 26 days, the patient was discharged without obvious symptoms. The presence of HHV-6A in the stored CSF sample, which was unexpectedly identified by mNGS, was further confirmed by real-time PCR following the protocol described by Gautheret-Dejean et al. (16).

\section{DISCUSSIONS}

Viral encephalitis accounts for $20-50 \%$ of microbiologically confirmed cases of encephalitis, with herpes simplex virus being the most common etiologic agent followed by VZV (17). Detection of the specific viral IgM antibodies or nucleic acid in CSF sample is regarded as a confirmation of viral encephalitis $(3,17)$. As for the VZV encephalitis, those parameters have variably diagnostic sensitivities, possibly due to different patients being enrolled in the studies (18-21). In a study on VZV CNS infections, VZV nucleic acid was detectable in the CSF only in one-fourth of the patients (18). Since CSF VZV viral load is correlated with the severity of VZV CNS infection $(22,23)$, 
TABLE 3 | Pathogens unexpectedly reported by the mNGS.

\begin{tabular}{|c|c|c|}
\hline & $\begin{array}{l}\text { No. of } \\
\text { positive CSF } \\
\text { sample }\end{array}$ & $\begin{array}{c}\text { No. of sequence } \\
\text { reads }\end{array}$ \\
\hline Cytomegalovirus & 5 & $1,4,5,7$, and 26 \\
\hline Herpes simplex virus- 1 & 5 & $1,1,4,8$, and 155 \\
\hline Human polyomavirus-5 & 2 & 3 and 3 \\
\hline Candida albicans & 2 & 4 and 10 \\
\hline Mycoplasma species & 2 & 3 and 5 \\
\hline $\begin{array}{l}\text { Others: human betaherpesvirus } 6 \text { A, } \\
\text { human papillomavirus- } 5 \text {, Staphylococcus } \\
\text { aureus, Rickettsia felis, Aspergillus } \\
\text { fumigatus, Torque teno virus } 28 \text {, human } \\
\text { papillomavirus-19, Epstein-Barr virus, and } \\
\text { molluscum contagiosum virus }\end{array}$ & $\begin{array}{l}1 \text { for each } \\
\text { pathogen }\end{array}$ & $\begin{array}{c}889,10,4,4,4,3,3,2 \\
\text { and } 2 \text {, respectively }\end{array}$ \\
\hline
\end{tabular}

CSF, cerebrospinal fluid.

patients with mild neurological involvement may have extremely low viral burden below the detection limit of VZV PCR. The presence of VZV IgM antibody in CSF could be analyzed by using the commercial ELISA kits. However, a CSF standard is generally not provided by the manufacturers, which makes setting a cutoff value complicated. This may be one of the reasons that contributes to the low utility of this parameter in diagnosing VZV CNS infection, although a study revealed a low sensitivity of CSF IgM antibody for identification of VZV CNS infection (18).

For the above-mentioned reasons, a negative CSF VZV IgM test or nucleic acid test does not necessarily rule out VZV CNS infection. Therefore, in the present study, we did not arbitrarily define "true positive" or "true negative" cases of VZV CNS infection. In this context, the exact sensitivity of each microbiological test for diagnosing of CNS infection was not calculated. Instead, the magnitude of positive result was compared. Our study showed that, compared with CSF VZV IgM antibody test or CSF VZV PCR test, CSF mNGS identified much higher number of cases with clues of VZV CNS infection. Of note, all the CSF samples with positive VZV PCR results tested positive for VZV sequence reads by using mNGS. Those findings suggest that CSF VZV-specific PCR may not improve the sensitivity of diagnosing VZV CNS infection when mNGS has already been performed on CSF samples.

The mNGS focuses on the whole genome sequence of the pathogen which is broken into small sequence segments that are unbiasedly amplified and measured. Therefore, sequences of a given pathogen are more likely to be detected by mNGS targeting the whole genome of the pathogen, as compared with pathogen-specific PCR which generally targets single or two regions of the genome sequence (24). Moreover, in the case of target gene mutation, pathogen-specific PCR may not be able to amplify the target gene, which leads to a false negative result. The abovementioned reasons could explain why, in our study, mNGS performed better with regard to the detection of VZV sequences compared with PCR methods. Finally, as shown in Table 1, the level of CSF white blood cells infiltration is relatively low. In this condition, the sensitivity of mNGS for pathogen detection may be less impacted by human genome contamination.
The main advantage of mNGS is unbiased sampling which enables broad identification of pathogens in a hypothesis-free manner (6). Using this relatively novel strategy, others and we have already diagnosed a number of unexpected or rare cases with infectious etiologies $(7,9,25-28)$. In the current study on patients with suspected VZV CNS infection, HHV-6A was unexpectedly identified by CSF mNGS from a patient with obvious neurological symptoms. Since HHV-6A has been shown to be related to neurological diseases (29-33), the presence of HHV-6A in the CSF sample of our patient could be of clinical importance. The finding re-emphasizes that agnostic mNGS is a useful strategy for identification of the etiologic agent when the treating physician is not familiar with the encountered clinical situation.

The mNGS is not a perfect diagnostic strategy in clinical microbiology. The unbiased sampling is not just an advantage but also, to some extent, could be a drawback. First, clinical samples are dominated by human host background. Unwanted human DNA occupies the majority of sequence reads of the mNGS which would limit the sensitivity of this strategy for pathogen identification $(6,34)$. Second, sequence reads from microorganisms not related to the clinical diseases can also be reported, which complicates the interpretation of the mNGS results. Those microbial contaminants, such as environmental microbes and skin flora, could be generated during sample collection and processing. Even the reagents used for processing may be contaminated by microbial DNA $(6,34)$. Finally, mNGS does not have the power to distinguish invasive infection from colonization. In addition, it could not differentiate between latent and active infections. In this condition, mNGS results should be interpreted based on the clinical judgement of the treating physicians.

As shown in Table 3, trace amounts of microbial nucleic acids, most commonly the CMV and HSV-1, were reported. CMV and HSV-1 are common human pathogens, both of which could cause CNS diseases (3). The mNGS, itself, could not answer whether those microbial DNAs really presented in the CSF samples. Additionally, it is not possible to tell whether those pathogens caused the neurological symptoms of our patients. Those drawbacks of mNGS are not likely to be completely resolved in the near future. At this point, it is advocated that mNGS results are treated as a list of candidate etiological agents which help to narrow the diagnosis. Only when the limitations of the mNGS are fully understood, then the clinicians may properly interpretate the mNGS results.

In conclusion, our study found that CSF mNGS was the most sensitive microbiological test for diagnosing VZV CNS infection, and could unexpectedly identify the causative agent that were not recognized based on traditional diagnostic tests. Perhaps, for the diagnoses of viral encephalitis caused by DNA virus, with continuing improving the methodology of mNGS, pathogenspecific PCR may not be needed when mNGS has already been used for pathogen detection. Nevertheless, pathogen-specific antibody detection may still be performed because it provides different biological information. Finally, although the sensitivity of mNGS can be further increased by technical innovation, the specificity will continue to be a great concern. Clinical judgement 
of the treating physicians is very important for interpretating the results.

\section{DATA AVAILABILITY STATEMENT}

The datasets used and/or analyzed during the current study are available from the corresponding author on reasonable request. The raw sequences of mNGS were deposited to the NCBI database under accession numbers PRJNA784638.

\section{ETHICS STATEMENT}

The studies involving human participants were reviewed and approved by the Second Hospital of Nanjing. Written informed consent to participate in this study was provided by the participants' legal guardian/next of kin.

\section{AUTHOR CONTRIBUTIONS}

$\mathrm{ZH}$ designed this study. $\mathrm{YZ}, \mathrm{CD}$, and $\mathrm{ZH}$ collected the data. MX, $\mathrm{WW}$, and $\mathrm{ZH}$ completed the experiment. MX, ZP, WC, and $\mathrm{ZH}$

\section{REFERENCES}

1. Somand D, Meurer W. Central nervous system infections. Emerg Med Clin North Am. (2009) 27:89-100. doi: 10.1016/j.emc.2008.07.004

2. Dubot-Pérès A, Mayxay $M$, Phetsouvanh $R$, Lee SJ, Rattanavong S, Vongsouvath $M$, et al. Management of central nervous system infections, vientiane, laos, 2003-2011. Emerg Infect Dis. (2019) 25:898-910. doi: 10.3201/eid2505.180914

3. Tunkel AR, Glaser CA, Bloch KC, Sejvar JJ, Marra CM, Roos KL, et al. The management of encephalitis: clinical practice guidelines by the Infectious Diseases Society of America. Clin Infect Dis. (2008) 47:303-27. doi: $10.1086 / 589747$

4. Granerod J, Cunningham R, Zuckerman M, Mutton K, Davies NW, Walsh $\mathrm{AL}$, et al. Causality in acute encephalitis: defining aetiologies. Epidemiol Infect. (2010) 138:783-800. doi: 10.1017/S0950268810000725

5. He T, Kaplan S, Kamboj M, Tang YW. Laboratory diagnosis of central nervous system infection. Curr Infect Dis Rep. (2016) 18:35. doi: 10.1007/s11908-016-0545-6

6. Gu W, Miller S, Chiu CY. Clinical metagenomic next-generation sequencing for pathogen detection. Ann Rev Pathol. (2019) 14:319-38. doi: 10.1146/annurev-pathmechdis-012418-012751

7. Hu Z, Weng X, Xu C, Lin Y, Cheng C, Wei H, et al. Metagenomic nextgeneration sequencing as a diagnostic tool for toxoplasmic encephalitis. Ann Clin Microbiol Antimicrob. (2018) 17:45. doi: 10.1186/s12941-0180298-1

8. Wilson MR, Sample HA, Zorn KC, Arevalo S, Yu G, Neuhaus J, et al. Clinical metagenomic sequencing for diagnosis of meningitis and encephalitis. N Engl J Med. (2019) 380:2327-40. doi: 10.1056/NEJMoa1803396

9. Fang M, Weng X, Chen L, Chen Y, Chi Y, Chen W, et al. Fulminant central nervous system varicella-zoster virus infection unexpectedly diagnosed by metagenomic next-generation sequencing in an HIV-infected patient: a case report. BMC Infect Dis. (2020) 20:159. doi: 10.1186/s12879-020-4872-8

10. Hong NTT, Anh NT, Mai NTH, Nghia HDT, Nhu LNT, Thanh TT, et al. Performance of metagenomic next-generation sequencing for the diagnosis of viral meningoencephalitis in a resource-limited setting. Open Forum Inf Dis. (2020) 7:ofaa046. doi: 10.1093/ofid/ofaa046

11. Xing X-W, Zhang J-T, Ma Y-B, He M-W, Yao G-E, Wang W, et al. Metagenomic next-generation sequencing for diagnosis of infectious encephalitis and meningitis: a large, prospective case series of 213 patients. Front Cell Infect Microbiol. (2020) 10:88. doi: 10.3389/fcimb.2020.00088 analyzed the data. BS, JC, and CC were involved in the clinical management of the patients and analyzed the clinical data. This manuscript was initially drafted by $\mathrm{YZ}, \mathrm{CD}$, and $\mathrm{ZH}$ and then revised by MX, ZP, WW, BS, JC, CC, WC, HW, and ZH. All authors approved the final manuscript.

\section{FUNDING}

This study was funded in part by the project of Jiangsu province medical youth talent (QNRC2016059) and the Nanjing medical science and technique development foundation (YKK18153). The funders had no role in study design, data collection and analysis, decision to publish, or preparation of the manuscript.

\section{SUPPLEMENTARY MATERIAL}

The Supplementary Material for this article can be found online at: https://www.frontiersin.org/articles/10.3389/fpubh. 2021.738412/full\#supplementary-material

12. Wang S, Chen Y, Wang D, Wu Y, Zhao D, Zhang J, et al. The feasibility of metagenomic next-generation sequencing to identify pathogens causing tuberculous meningitis in cerebrospinal fluid. Front Microbiol. (2019) 10:1993. doi: 10.3389/fmicb.2019.01993

13. Yan L, Sun W, Lu Z, Fan L. Metagenomic Next-Generation Sequencing (mNGS) in cerebrospinal fluid for rapid diagnosis of Tuberculosis meningitis in HIV-negative population. Int J Inf Dis. (2020) 96:270-5. doi: 10.1016/j.ijid.2020.04.048

14. Ji X-C, Zhou L-F, Li C-Y, Shi Y-J, Wu M-L, Zhang Y, et al. Reduction of human DNA contamination in clinical cerebrospinal fluid specimens improves the sensitivity of metagenomic next-generation sequencing. $\mathrm{J} \mathrm{Mol}$ Neurosci. (2020) 70:659-66. doi: 10.1007/s12031-019-01472-z

15. Persson A, Bergström T, Lindh M, Namvar L, Studahl M. Varicella-zoster virus CNS disease-viral load, clinical manifestations and sequels. J Clin Virol. (2009) 46:249-53. doi: 10.1016/j.jcv.2009.07.014

16. Gautheret-Dejean A, Manichanh C, Thien-Ah-Koon F, Fillet AM, Mangeney $\mathrm{N}$, Vidaud $\mathrm{M}$, et al. Development of a real-time polymerase chain reaction assay for the diagnosis of human herpesvirus- 6 infection and application to bone marrow transplant patients. J Virol Methods. (2002) 100:27-35. doi: 10.1016/S0166-0934(01)00390-1

17. Tyler KL. Acute viral encephalitis. N Engl J Med. (2018) 379:557-66. doi: 10.1056/NEJMra1708714

18. Koskiniemi M, Piiparinen $\mathrm{H}$, Rantalaiho $\mathrm{T}$, Eränkö P, Färkkilä $M$, Räihä K, et al. Acute central nervous system complications in varicella zoster virus infections. J Clin Virol. (2002) 25:293-301. doi: 10.1016/S1386-6532(02)00020-3

19. Gregoire SM, Van Pesch V, Goffette S, Peeters A, Sindic CJ. Polymerase chain reaction analysis and oligoclonal antibody in the cerebrospinal fluid from 34 patients with varicella-zoster virus infection of the nervous system. J Neurol Neurosurg Psychiatry. (2006) 77:938-42. doi: 10.1136/jnnp.2006.090316

20. Nagel MA, Cohrs RJ, Mahalingam R, Wellish MC, Forghani B, Schiller A, et al. The varicella zoster virus vasculopathies: clinical, CSF, imaging, and virologic features. Neurology. (2008) 70:853-60. doi: 10.1212/01.wnl.0000304747.38502.e8

21. De Broucker T, Mailles A, Chabrier S, Morand P, Stahl J-P. Acute varicella zoster encephalitis without evidence of primary vasculopathy in a case-series of 20 patients. Clin Microbiol Inf. (2012) 18:808-19. doi: 10.1111/j.1469-0691.2011.03705.x

22. Aberle SW, Aberle JH, Steininger C, Puchhammer-Stöckl E. Quantitative real time PCR detection of Varicella-zoster virus DNA in cerebrospinal fluid in 
patients with neurological disease. Med Microbiol Immunol. (2005) 194:7-12. doi: 10.1007/s00430-003-0202-1

23. Rottenstreich $\mathrm{A}, \mathrm{Oz} \mathrm{ZK}$, Oren I. Association between viral load of varicella zoster virus in cerebrospinal fluid and the clinical course of central nervous system infection. Diagn Microbiol Infect Dis. (2014) 79:174-7. doi: 10.1016/j.diagmicrobio.2014.02.015

24. Metzker ML. Sequencing technologies-the next generation. Nat Rev Genet. (2010) 11:31-46. doi: 10.1038/nrg2626

25. Mongkolrattanothai K, Naccache SN, Bender JM, Samayoa E, Pham E, Yu G, et al. Neurobrucellosis: unexpected answer from metagenomic next-generation sequencing. J Pediatric Infect Dis Soc. (2017) 6:393-8. doi: 10.1093/jpids/piw066

26. Brown JR, Bharucha T, Breuer J. Encephalitis diagnosis using metagenomics: application of next generation sequencing for undiagnosed cases. $J$ Infect. (2018) 76:225-40. doi: 10.1016/j.jinf.2017.12.014

27. Huang Z, Zhang C, Li W, Fang X, Wang Q, Xing L, et al. Metagenomic nextgeneration sequencing contribution in identifying prosthetic joint infection due to Parvimonas micra: a case report. J Bone Joint Inf. (2019) 4:50-5. doi: 10.7150/jbji.30615

28. Jin W, Miao Q, Wang M, Zhang Y, Ma Y, Huang Y, et al. A rare case of adrenal gland abscess due to anaerobes detected by metagenomic next-generation sequencing. Ann Transl Med. (2020) 8:123. doi: 10.21037/atm.2020.01.123

29. Asano Y, Yoshikawa T, Kajita Y, Ogura R, Suga S, Yazaki T, et al. Fatal encephalitis/encephalopathy in primary human herpesvirus-6 infection. Arch Dis Child. (1992) 67:1484-5. doi: 10.1136/adc.67.12.1484

30. Ogata M, Satou T, Kawano R, Takakura S, Goto K, Ikewaki J, et al. Correlations of HHV-6 viral load and plasma IL-6 concentration with HHV-6 encephalitis in allogeneic stem cell transplant recipients. Bone Marrow Transplant. (2010) 45:129-36. doi: 10.1038/bmt.2009.116

31. Ogata M, Satou T, Kadota J-I, Saito N, Yoshida T, Okumura H, et al. Human Herpesvirus 6 (HHV-6) reactivation and HHV-6 encephalitis after allogeneic hematopoietic cell transplantation: a multicenter, prospective study. Clin Infect Dis. (2013) 57:671-81. doi: 10.1093/cid/cit358

32. Leibovitch EC, Jacobson S. Evidence linking HHV-6 with multiple sclerosis: an update. Curr Opin Virol. (2014) 9:127-33. doi: 10.1016/j.coviro.2014. 09.016

33. Eliassen E, Hemond CC, Santoro JD. HHV-6-associated neurological disease in children: epidemiologic, clinical, diagnostic, and treatment considerations. Pediatr Neurol. (2020) 105:10-20. doi: 10.1016/j.pediatrneurol.2019.10.004

34. Simner PJ, Miller S, Carroll KC. Understanding the promises and hurdles of metagenomic next-generation sequencing as a diagnostic tool for infectious diseases. Clin Infect Dis. (2018) 66:778-88. doi: 10.1093/ $\mathrm{cid} / \mathrm{cix} 881$

Conflict of Interest: The authors declare that the research was conducted in the absence of any commercial or financial relationships that could be construed as a potential conflict of interest.

Publisher's Note: All claims expressed in this article are solely those of the authors and do not necessarily represent those of their affiliated organizations, or those of the publisher, the editors and the reviewers. Any product that may be evaluated in this article, or claim that may be made by its manufacturer, is not guaranteed or endorsed by the publisher.

Copyright (c) 2022 Zhu, Xu, Ding, Peng, Wang, Sun, Cheng, Chen, Chen, Wei and $\mathrm{Hu}$. This is an open-access article distributed under the terms of the Creative Commons Attribution License (CC BY). The use, distribution or reproduction in other forums is permitted, provided the original author(s) and the copyright owner(s) are credited and that the original publication in this journal is cited, in accordance with accepted academic practice. No use, distribution or reproduction is permitted which does not comply with these terms. 